\title{
ON THE HESSIAN OF THE CARATHÉODORY METRIC
}

\section{JACOB BURBEA}

Abstract. The generalized lower Hessian of an upper semi-continuous function $f$ near a point $z$ in $\mathbf{C}^{n}$ is introduced (for $n=1$ see Heins, Nagoya Math. J. 21 (1962), 1-60). With this we introduce a "sectional curvature" and we prove that the sectional curvature of the Caratheodory-Reiffen metric is always $\leqq-4$. This generalizes a result of Suita (Kodai Math. Sem. Rep. 25 (1973), 215-218) in the one dimensional case. The sectional curvatures of the ball and polydisk are always -4 . A few other properties of the Hessian of the above metric are shown.

1. Preliminaries. For a point $z=\left(z_{1}, \cdots, z_{n}\right) \in \mathbf{C}^{n}$, we set $\|z\|$ $=\left(\sum_{j=1}^{n}\left|z_{j}\right|^{2}\right)^{1 / 2}$ and for $a \in \mathbf{C}^{n}, \quad r>0, \quad B(a, r)=\left\{z \in \mathbf{C}^{n}\right.$ : $\|z-a\|<r\}$ denotes the open ball centered at $a$ and with radius $r$. The natural pairing between a contangent vector $\alpha$ and a tangent vector $v$ is denoted by $\langle\alpha, v\rangle$. Especially, if $f$ is a $C^{1}$ function near the point $z$, and $v=\left(v_{1}, \cdots, v_{n}\right) \in \mathbf{C}^{n}$, then

$$
\langle\partial f(z), v\rangle=\sum_{j=1}^{n} \frac{\partial f}{\partial z_{j}} v_{j} .
$$

Let $D$ be a bounded domain in $C^{n}$ and let $U$ be the unit disk in C. $H(D: U)$ designates the family of holomorphic functions from $D$ into $U$. For fixed $\zeta$ in $D$ we write $H_{\zeta}(D: U)=\{f \in H(D: U): f(\zeta)$ $=0\}$. For each $\zeta \in D, C_{D}(\zeta ;)$ is the function defined on the complex tangent space of $D$ at $\zeta$ by

$$
C_{D}(\zeta ; v)=\sup \{|\langle\partial f(\zeta), v\rangle|: f \in H(D: U)\}
$$

(cf. Reiffen [3]). Evidently,

$$
C_{D}(\zeta ; v)=\sup \left\{|\langle\partial f(\zeta), v\rangle|: f \in H_{\zeta}(D: U)\right\} .
$$

$C_{D}$ is called the Carathéodory metric for $D$. Since $H(D: U)$ is a normal family, the supremum in the definition of $C_{D}(\zeta ; v)$ is attained by some $F \in H_{\zeta}(D: U)$. Here $F(z)=F(z ; \zeta, v)$. By a normal family argument $C_{D}(\zeta: v)$ is continuous in $(\zeta, v)$. For the above mentioned properties see [3]. Moreover, $C_{D}(\zeta ; v)$ is a locally Lipschitz function [1].

Received by the editors on July 19,1976, and in revised form on November 8 , 1976.

AMS (MOS) 1970 Subject Classifications. 32H15, 32A30.

Key words and phrases. hessian, Carathéodory metric, curvature. 
We also note that the Carathéodory metric has the "distance-decreasing" property that is, if $\phi: D \rightarrow D^{*}$ is a holomorphic mapping, then $C_{D}\left(\phi(z) ; \phi^{*}(v)\right) \leqq C_{D^{*}}(z ; v)$. Here $\left[\phi^{*}(v)\right]_{j}=\sum_{k=1}\left(\partial \phi_{j} / \partial z_{k}\right) v_{k}, j=1$, $\cdots, n$. Hence $C_{D}$ is invariant under biholomorphic mappings. Clearly the Carathéodory metric for the unit disk $U$ is given by

$$
C_{U}(z ; v)=\frac{|v|}{1-|z|^{2}} .
$$

REMarK: There is no essential loss of generality in restricting our attention to bounded domains. The Carathéodory metric may be defined on arbitrary complex manifolds, although it may be zero in some directions $v$.

We have $C_{D}(z: v) \geqq\|v\| / \delta_{D}$, where $\delta_{D}$ is the radius of the smallest ball surrounding $D$. On the other hand:

Proposition 1. $C_{D}(z: v) \leqq\|v\| / d(z)$, where $d(z)$ is the distance of $z \in D$ from the boundary of $D$.

Proof. Consider the ball $B(z, r) \subset D$ and let $f \in H_{z}(D: U)$. Let $v \in C^{n}-\{0\}$ be fixed. The function $\phi(w)=f(z+w v)$ maps the disk $|w|<r \mid\|v\|$ into the unit disk $U$ and $\varphi(0)=0$. Hence, by Schwarz's lemma, $\left|\varphi^{\prime}(0)\right| \leqq\|v\| / r$. But $\varphi^{\prime}(0)=\langle\partial f(z), v\rangle$ and so $|\langle\partial f(z), v\rangle| \leqq\|v\| / r$. Put $r=d(z)$ and the assertion follows.

Proposition 2. Let $v \in \mathrm{C}^{n}-\{0\}$ be fixed. Then $\log C_{D}(z ; v)$ is plurisubharmonic in $z \in D$.

Proof. Recall that if $\exists$ is a family of plurisubharmonic functions which are locally uniformly bounded from above, then $\varlimsup_{z^{\prime} \rightarrow z^{\prime}} \sup _{g \in \mathcal{g}} g\left(z^{\prime}\right)$ is plurisubharmonic. Let $\ni=\{\log |\langle\partial f, v\rangle|: f$ $\left.\in H_{z}(D: U)\right\}$. Clearly, the members of $\exists$ are plurisubharmonic and, as in the proof of Proposition $1, \log |\langle\partial f(z), v\rangle| \leqq \log \|v\|-\log r$ in the ball $B(z, r) \subset D$. Thus, since $\log C_{D}(z ; v)$ is continuous, $\log C_{D}(z ; v)=\sup _{g \in 9} g(z)$ is plurisubharmonic.

2. The Hessian. Let $f$ be upper semi-continuous near $z \in \mathrm{C}^{n}$ and let $u \in C^{n}-\{0\}$. The generalized lower Hessian (or "Laplacian") of $f$ at $z$ along the direction $u$ is defined by

$$
\Delta_{u} f(z)=4 \lim _{r \rightarrow 0} \frac{1}{r^{2}}\left(\frac{1}{2 \pi} \int_{0}^{2 \pi} f\left(z+r e^{i \theta} u\right) d \theta-f(z)\right)
$$

(cf. also Heins [2]). Note that, if $f$ is a $C^{2}$ function near $z$, then $\Delta_{u} f(z)$ reduces to four times the usual Hessian of $f$ at $z$ along $u$, that is 


$$
\Delta_{u} f(z)=4 \sum_{i, j=1}^{n} \frac{\partial^{2}}{\partial z_{i} \partial \bar{z}_{j}} f(z) u_{i} \bar{u}_{j} .
$$

In this case, if also $n=1$, then $\Delta_{u} f(z)=\Delta f(z)|u|^{2}$, where $\Delta$ is the usual Laplacian. Clearly, if $f$ assumes a local minimum at $z$ then $\Delta_{u} f(z) \geqq 0$ for each direction $u$.

Let $v \in C^{n}-\{0\}$ and consider $F(z)=F(z: \zeta, v)$ as before. Define

$$
\lambda(z ; u)=\frac{|\langle\partial F(z), u\rangle|}{1-|F(z)|^{2}} .
$$

Therefore,

$$
\log \lambda(z ; u)=\log |\langle\partial F(z), u\rangle|-\log (1-F(z) \overline{F(z)}) .
$$

The first term on the right is pluriharmonic and hence its Hessian along any direction (independently of $u$ ) is zero.

Consequently,

$$
\Delta_{w} \log \lambda(z ; u)=4 \lambda(z ; w)^{2}
$$

for each direction $w \in C^{n}$ and whenever $\langle\partial F(z), u\rangle \neq 0$. Especially

$$
\Delta_{w} \log \lambda(\zeta ; v)=4 \lambda(\zeta ; w)^{2} .
$$

Note that $\lambda(\zeta ; v)=C_{D}(\zeta ; v)$.

The following theorem is the $n$-dimensional generalization of Suita's result [5].

Theorem 1. Let $\zeta \in D$ and $v \in \mathrm{C}^{n}-\{0\}$ be fixed. Then

$$
\Delta_{u} \log C_{D}(\zeta ; v) \geqq 4 \lambda(\zeta ; u)^{2}
$$

for each direction $u \in \mathrm{C}^{n}$ and thus again $\log C_{D}(\zeta ; v)$ is plurisubharmonic.

Proof. We have $C_{D}(\zeta ; v)=|\langle\partial F(\zeta), v\rangle|$. Set

$$
G(z)=\frac{F(z)-F(\eta)}{1-\overline{F(\eta)} F(z)}
$$

Thus $G \in H_{\eta}(D ; U)$ and therefore $|\langle\partial G(\eta), u\rangle| \leqq C_{D}(\eta ; u)$ for each direction $u$. However, $|\langle\partial G(\eta), u\rangle|=\lambda(\eta ; u)$. Thus $\lambda(\eta ; v) \leqq$ $C_{D}(\eta ; v)$ and we have equality at $\eta=\zeta$. Near $\zeta, \lambda(\eta ; v)$ is positive and hence $\log C_{D}(\eta ; v) / \lambda(\eta ; v)$ assumes a local minimum at $\eta=\zeta$ and so

$$
\Delta_{u} \log C_{D}(\zeta ; v) \geqq \Delta_{u} \log \lambda(\zeta ; v)
$$

for each direction $u$. The theorem now follows from (2.1).

Let $v \in C^{n}-\{0\}$ and assume that the metric $\mu(z ; v)$ is a positive 
upper semi-continuous function at $z$. The "curvature" of $\mu(z ; v)$ at $z$ in the direction $v$ is given by

$$
\mathcal{K}(\mu: z, v)=-\frac{1}{\mu(z ; v)^{2}} \Delta_{v} \log \mu(z ; v) .
$$

By (2.1) the "curvature" of $\lambda(z ; v)$ is -4 at $z=\zeta$.

THEOREM 2. The "curvature" of $C_{D}(\zeta ; v)$ is always $\leqq-4$.

Proof. By Theorem $1, \Delta_{v} \log C_{D}(\zeta ; v) \geqq 4 \lambda(\zeta ; v)^{2}$ and, since $\lambda(\zeta ; v)$ $=C_{D}(\zeta ; v)$, the assertion follows.

The above theorem was first proved by Suita [5] in the case of $n=1$.

\section{Examples.}

Example 1. Consider the polydisk $D=\left\{\left(z_{1}, \cdots, z_{n}\right):\left|z_{j}\right|<1,1 \leqq\right.$ $j \leqq n\}$. Then

$$
C_{D}(z ; v)=\max \left(\frac{\left|v_{1}\right|}{1-\left|z_{1}\right|^{2}}, \cdots, \frac{\left|v_{n}\right|}{1-\left|z_{n}\right|^{2}}\right) .
$$

Here $C_{D}(z ; v)$ is continuous but obviously not a $C^{1}$-function.

Although expression (3.1) is a consequence of a general theorem on product domains (cf. Royden [4]) it is nevertheless instructive to prove it here directly.

Since $D=U \times \cdots \times U$ ( $n$ copies of the unit disk), it is plain that $C_{D}(z ; v) \geqq C_{U}\left(z_{j} ; v_{j}\right), 1 \leqq j \leqq n$. In fact, let $\phi_{j}: D \rightarrow U$ be given by $\phi_{j}(z)=z_{j}$. Then, using the distance-decreasing property, $C_{D}(z ; v) \geqq$ $C_{U}\left(\phi_{j}(z) ; \phi_{j}^{*}(v)\right)=C_{U}\left(z_{j} ; v_{j}\right)$. On the other hand we may assume, because of the homogeneity of $D$, that $z=0$ and $\left|v_{1}\right|=\max \left(\left|v_{1}\right|, \cdots\right.$, $\left.\left|v_{n}\right|\right)>0$. Consider the holomorphic mapping $\phi: U \rightarrow D$ defined by

$$
\phi\left(z_{1}\right)=\left(z_{1}, \frac{v_{2}}{v_{1}} z_{1}, \cdots, \frac{v_{n}}{v_{1}} z_{1}\right) .
$$

A second application of the distance-decreasing property and a use of $(1.1)$ show that

$$
C_{D}(0 ; v)=C_{D}\left(\phi(0) ; \phi^{*}\left(v_{1}\right)\right) \leqq C_{U}\left(0 ; v_{1}\right)=\left|v_{1}\right|
$$

and hence (3.1) follows.

We now show that the "curvature" of this metric is always -4 . This, of course, needs to be proven only for $z=0$. We may assume that $C_{D}(0 ; v)=\max \left(\left|v_{1}\right|, \cdots,\left|v_{n}\right|\right)=\left|v_{1}\right|$. A simple calculation then shows that 


$$
C_{D}\left(r e^{i \theta} v ; v\right)=\left|v_{1}\right| /\left(1-r^{2}\left|v_{1}\right|^{2}\right)
$$

for a sufficiently small $r$. An application of L'Hospital's rule gives

$$
\begin{aligned}
\Delta_{v} \log C_{D}(0 ; v) & =4 \lim _{r \rightarrow 0} \frac{1}{r^{2}}\left(\frac{1}{2 \pi} \int_{0}^{2 \pi} \log \frac{\left|v_{1}\right|}{1-r^{2}\left|v_{1}\right|^{2}} d \theta-\log \left|v_{1}\right|\right) \\
& =4 \lim _{r \rightarrow 0} \frac{1}{r^{2}} \log \left(1-r^{2}\left|v_{1}\right|^{2}\right)=4\left|v_{1}\right|^{2} .
\end{aligned}
$$

Hence $\Delta_{v} \log C_{D}(\zeta ; v)=4 C_{D}(\zeta ; v)^{2}$ as asserted.

Example 2. Consider the ball $D=\left\{z \in C^{n}:\|z\|<1\right\}$. Then (cf. [3])

$$
C_{D}(z ; v)^{2}=\frac{\|v\|^{2}}{1-\|z\|^{2}}+\frac{|(z, v)|^{2}}{\left(1-\|z\|^{2}\right)^{2}},
$$

where $(z, v)=\sum_{j=1}^{n} z_{j} \bar{v}_{j}$. Here $C_{D}(z ; v)$ is a real analytic function. The "curvature" is always -4. Again, it suffices to show this for $z=0$. In this case $C_{D}(0 ; v)=\|v\|$ and

$$
C_{D}\left(r e^{i \theta} v ; v\right)=\frac{\|v\|}{1-r^{2}\|v\|^{2}}
$$

Thus, using L'Hospital's rule,

$$
\begin{aligned}
\Delta_{v} \log C_{D}(0 ; v) & =-4 \lim _{r \rightarrow 0} \frac{1}{2 \pi r^{2}} \int_{0}^{2 \pi} \log \left(1-r^{2}\|v\|^{2}\right) d \theta \\
& =4\|v\|^{2}=4 C_{D}(0 ; v)^{2} .
\end{aligned}
$$

\section{Bibliography}

1. C. J. Earle and R. S. Hamilton, A fixed point theorem for holomorphic mappings, Global Analysis, Proc. of Symposium in Pure Math. 16, Amer. Math. Soc., Providence, 1965.

2. M. Heins, On a class of conformal metrics, Nagoya Math. J. 21 (1962), $1-60$.

3. H. J. Reiffen, Die differentialgeometrischen Eigenschaften der invarianten Distanzfunktion von Carathéodory, Schrift. Math. Inst. Univer. Münster, No. 26 (1963).

4. H. L. Royden, Remarks on the Kobayashi metric, Springer-Verlag Lecture Notes in Math. 185 (1971), 125-137.

5. N. Suita, On a metric induced by analytic capacity, Kodai Math. Sem. Rep. 25 (1973), 215-218.

Department of Mathematics, University of Pittsburgh, Pittsburgh, Pennsylvania 15260 
' 